\title{
A Methodological Approach for Optimum Preservation Results: The Packaging Paradigm
}

\author{
Antonis Kanavouras ${ }^{a^{*}}$ And Frank A. Coutelieris ${ }^{b}$ \\ ${ }^{a}$ Department of Food Science and Human Nutrition, Agricultural University of Athens, 55 Iera Odos Str., \\ GR-11855, Athens, Greece \\ b Department of Environmental \& Natural Resources Management, School of Engineering, University of Patras, \\ 2 Seferi Str., GR-30100 Agrinio, Greece \\ ${ }^{*}$ Corresponding author \\ antonios.kanavouras@aua.gr \\ TEL: +302105294738
}

Received: 08 September 2016; Published online: 18 April 2017

Invited paper from the $4^{\text {th }}$ International ISEKI_Food Conference - ISEKI_Food 2016 - Bridging Training and Research for Industry and the Wider Community - Responsible Research and Innovation in the Food Value Chain

\begin{abstract}
The food preservation hypothesis as impacted by overall packaging applications is considered in this work. The objective was to devise a decision supportive method for the selection of "just-right" packaging materials, techniques and procedures. For that, food preservation was critically approached in order to identify the optimum outcome at experimental and packaging selection decision-making levels. A mathematically supported and proven knowledge classification, and the establishment of a straightforward coherence mode among the principles of the natural systemic phenomena, were used. The ultimate aim of this work was to justifiably surpass a simple description of packaging according to its measurable specifications, and instead, engage its inherent properties into a cyclic 8-steps-process for eventually understanding its potential to support any particular preservation hypothesis in question. The proposed methodology includes primarily, the consideration of the study hypothesis and, in parallel, the conclusive remarks and claims with respect to the experimental factors involved (properties, parameters, relations and conditions). Considering the experimentally controlled set-ups that a researcher has to expose the food system to and the role of packaging in obtaining its preservation potential, our method supports the experimenters in selecting the experimental conditions under which the preservation hypothesis can be disclaimed and furthermore, it could indicate the way to reduce experimentation research waste.
\end{abstract}

Keywords: Shelf life; Packaging; Preservation; Engineering; Experimentation

\section{Introduction}

Trends in the modern food industry include the development of new and/or improved packaging materials. Consumer demands for more natural foods, and also for environmental protection, catalyzed during the past decades, support such developments. Active, intelligent and edible pack- aging are emerging based on multiple physical laws (Campos, Gerschenson, \& Flores, 2011). The preservation of a complex and potentially unpredictable system of "food-packagingenvironment" has been studied via quite a few variable practical ways. The majority of the most recent scientific research and development fields are still active today (Galic, Scetar, \& Kurek, 
2011; Raso \& Barbosa-Canovas, 2003; Senorans, Ibanez, \& Cifuentes, 2003; Gimenez, Ares, \& Ares, 2012). That could be due to an ever increasing emergence of needs, broadening of interests, complexity in defining quality or a lack of integrating the knowledge gained for optimum results.

Modern food packaging has made great advances as a result of global trends and consumer preferences. These advances aim to improve food quality and safety. Moreover, with the move toward globalization, food packaging is required to contribute to longer shelf life and the monitoring of safety and quality based upon international standards. The main packaging research on methodologies, applied for food preservation studies, may reveal their role and impact on the accumulated knowledge that could further support a more methodologically systematic approach and a potential higher efficiency in packaging experimentation. New technologies (such as nanotechnology) can address many of these requirements, and extend and implement the principal packaging functions - containment, protection and preservation, marketing and communications (Silvestre, Duraccio, \& Cimmino, 2011). Within developing areas of packaging, the permeability to gases and water vapor of the packaging films at the specific conditions of use, i.e. temperature, relative humidity and pressure gradient, are much needed for selecting the optimum packaging system to ensure the required shelf-life of the packed product. Migration from and absorption by plastic films also play an important role in this selection (Miltz, Passy, \& Mannheim, 1992). Packaging studies are reported in the literature for interrelated factors influencing the shelf life and keeping quality of meat products, specifically holding temperature, atmospheric oxygen $\left(\mathrm{O}_{2}\right)$, endogenous enzymes, moisture (dehydration), light and, most importantly, micro-organisms. The most investigated new preservation technologies for fresh meat are non-thermal inactivation technologies, including new packaging systems such as modified atmosphere packaging (MAP) and active packaging (AP) (Zhou, Xu, \& Liu, 2010; Gomez \& Lorenzo, 2012; Celia Resconi et al., 2012; Leygonie, Britz, \& Hoffman, 2011; Christophersen, Bertelsen, Andersen, Knuthsen, \& Skibsted, 1992). Similarly, the sensitivity of juices to oxidation through packaging indicated the evolution of dissolved oxygen was related to the ascorbic acid degradation, when exposed to high intensity light and regardless of the package permeability. Accordingly, changes in the aromatic profile of the juice were observed due to the degradation of terpenes to off-flavors (Bacigalupi et al., 2013). A mechanistic model to predict the shelf life of bottled orange juice, coupling $\mathrm{O}_{2}$ transfer and the ascorbic acid oxidation reaction, confirmed that oxygen permeation through the packaging material could not be neglected. Simulations confirmed that permeation through the packaging material, and especially through the closure, need to be considered and that mechanisms of ascorbic acid degradation must be better characterized in order to propose more accurate predictions. This model was able to predict the shelf life of orange juice with less than $20 \%$ error and, used in a reverse manner, is able to identify the window of $\mathrm{O}_{2}$ permeability required for maintaining a minimal level of ascorbic acid (chosen by the user) (Bacigalupi et al., 2013). A mathematical model that combines oxygen uptake from the outside environment with oxygen consumption by oxidative reactions, in a liquid packed food during storage, was developed for parameters such as the oxygen mass transfer coefficient and the rate constant of consumption reactions that were estimated by fitting the model to the experimental data. The influence of temperature on the reaction rate was well described by an Arrhenius type equation, from which the activation energy may derive (Ahrne et al., 1997).

Design of modified atmosphere packaging for fresh-cut produce, to be stored at a constant to varying temperatures, signified the effect of temperature on produce respiration and respectively described film permeability by an Arrhenius type of equation and the effect of $\mathrm{O}_{2}$ and $\mathrm{CO}_{2}$ on respiration rate by a Michaelis-Menten kinetic. Prediction and validation of an integrated approach showed that optimum conditions could be generated but an overestimation of the internal $\mathrm{O}_{2}$ concentrations occurred, probably because of the unconsidered $\mathrm{O}_{2}$ consumption of higher microbial activity at these examples of temperature abuse, with the risk of anoxic conditions to start at elevated temperatures (Jacxsens, Devlieghere, 
De Rudder, \& Debevere, 2000). Shelf life of minimally processed vegetables is often calculated by using the kinetic parameters of the Gompertz equation (Zwietering, Rombouts, \& Van 't Riet, 1993). The goodness-of-fit of the equation can be tested by using mesophilic bacteria cell loads from different minimally processed vegetables (packaged fresh-cut lettuce, fennel and shredded carrots) that differed for some process operations or for package atmosphere. An improved equation should be able to describe the data well and to estimate the shelf life. A kinetic modeling of the effect of storage temperature on the quality and shelf life of chilled fish, modified atmosphere-packed (MAP), used water loss, solid gain, salt content, and water activity throughout the shelf life study and treatment conditions. Quality assessment and temperature dependence for quality loss rates were modeled by the Arrhenius equation, validated under dynamic conditions by Tsironi and Taoukis (2010). Standard oxygen diffusion equations can be used to model oxygen transport within shelf-stable bottled food, with progress being made by consideration of two dimensional and three dimensional axisymmetric model problems. Realistic values for the diffusivity of oxygen inside food and different packaging materials, as well as the effects of a puncture on the nearby concentration profile and of a barrier with varying thickness are of interest in packaging research. The concentration is greatly affected by the geometry of any corners, and this could be further accentuated by variations in bottle thickness (Hall \& Otto, 1997).

Packaging can be an important factor in extending the shelf life of cereal-based goods (toast, frozen products, biscuits, cakes, pastas). Texture changes and flavor loss which manifest over the shelf life of a soft-baked good can usually be minimized or delayed by effective use of packaging materials, however, the extension of shelf life is proposed to be application specific (Galic et al., 2011).

Moreover, light-induced degradation reactions, such as in the case of milk products, may give rise to off-flavors, decrease the nutritional quality, and increase the speed and severity at which these phenomena develop. Packages need to provide a light barrier which is sufficient enough to avoid the photo oxidation during extended storage (Mestdagh, De Meulenaer, De Clippeleer, Devlieghere, \& Huyghebaert, 2005). Moisture permeability was also studied using an empirical kinetic model, considering both water transfer from fruit and water production as a result of Maillard processes (Miranda, Berna, Bon, \& Mulet, 2011).

By simulation, it was possible to assess the influence of a bottle's shape and size on the quality decay kinetics of virgin olive oil bottled in glass and plastic containers. It was shown that the quality decay kinetics of bottled virgin olive oil greatly depend on container geometry. However, the extent to which the container's geometrical factors affect the quality decay kinetics depends on the material used to make the bottle, and on the initial value of the oxygen partial pressure in the bottle headspace (Del Nobile, Bove, La Notte, \& Sacchi, 2003). The kinetic modeling of packed olive oil oxidation was studied (Kanavouras, Hernandez-Munoz, Coutelieris, \& Selke, 2004) and the activation energy for the production of oxidative markers, such as hexanal, for the prediction of the shelf life was further calculated (Coutelieris \& Kanavouras, 2005). Based on these outcomes, the researchers developed a mathematical model to estimate the probability of not reaching the end of its shelf life within certain time frames for olive oil packed in various packaging materials, when stored at multiple combinations of storage conditions (Kanavouras \& Coutelieris, 2006).

These few, but characteristic examples of foodpackaging studies for the preservation of various products, have certain commonalities, including the need for generalizing and standardizing the predictive efficiency of experimentation, either via simple deterministic processes or through advanced mathematical treatments for prediction improvements. We wish to propose that researchers should consider packaging as an adequate indicator for food systems. Packaging constitutes the natural barrier, up to the point that the environment starts, as well as the mass and energy transfer time-restrictor, due to its inherent properties and the intra-relationships among packaging, food and the environment surrounding the food. In that sense, a total foodpackaging development is the detailed justified 
The distinct role of packaging on food preservation $\mid 59$

engineering of the "packaging-as-an-indicator" functionality, considering both the food, as well as the environment, in terms of their optimum needs and the corresponding potential hazards. Having established the concept of packaging as a sub-system within the product, it would then be possible to view and handle packaging as an entity, i.e. as an independent system with its own inputs and outputs. For that, it is essential to ensure a packaging output that functions in time and place, influences the rest of the elements in the system and overall contributes to assured acceptable quality levels of the product.

From this view point, packaging becomes a risklevel-indicator to be fully described through its specifications. These specifications need to derive from appropriately designed experiments which aim to evaluate and manage the potential risks. To define the "just-right" packaging specifications, researchers have to incorporate into the experiments those particular conditions under which certain dependable packaging properties will be expressed. Then, by controlling these conditions researchers may eventually control the possible expression of the overall systemic properties. Accordingly, the selection of the packaging-indicator expressing conditions cannot be simply defined as an arbitrary set of experimental design factors but must be based on the rational process of incorporating the most efficient ones which allow the systemic phenomena to evolve, i.e. through a well-defined experimentation technology.

The objectives of this study may now be summarized as developing a methodology to express the potential impact of packaging on the possibility of disclaiming the food preservation hypothesis. The aim being that the methodology framework should include a knowledge classification-based approach. Food preservation case studies are also presented in order to demonstrate the use of this methodology in estimating the experimental conditions for defining the packaging specifications. It has been our goal to show through the case studies the need for potentially integrating those research parameters, as well as the alternative packaging selections that need to be considered for obtaining the maximum preservation outcome.

\section{Materials and Methods}

In general, systems such as packed foods possess a number of intrinsically coherent properties. In order to tackle such systems, we encourage a proper handling of the "theories-technologytechnique" triptych. It is this combination that will fully uncover and let us categorize the existing knowledge and reveal any potential gaps. Direct inter- and intra-developmental procedures among these areas of knowledge, as shown in Figure 1, initiated developmental outcomes from each of the above sources of empiricism. Similarly, engineering has theoretical, technological or technical sources of origin within sequential developments (Mitcham, 1994). This implies that the region where the three areas meet and overlap consists the pure and applied zone for paradigms in each system.

To fulfil the objectives of this work, we shall work on a cyclic approach, based on the fact that on every cycle each and every peripheral experience has the same equal distance from the "center". At the center of the cycle of experiences, the knowledge regarding each field may be positioned. Such a center, named as the "dominating example", is usually established and commonly accepted, through years of empirical research and cognitional understanding, being constantly subjected to revisions, criticism and reconstructions until there are more problems rather than answers emerging from research in that field. Eventually, research will lead to a so called "scientific revolution" that through the new knowledge shall establish a "new example" (Kuhn, 1962).

Accordingly, this present work will implement the systemic characteristics, predicates, attributes, qualities or properties as part of the systemic experiences that control the evolution of the phenomena within a given system, placed at a particular environment. Furthermore, it also wishes to propose a comprehensive methodology for fully meeting the research requirements. As a methodological consequence, conclusions of the research and preservation studies should focus on determining packaging (as means, processes and storage conditions) for satisfaction of the preservation hypothesis. Moreover, the particular systemic knowledge classes, as recorded in the existing knowledge, need to be classified in order to 


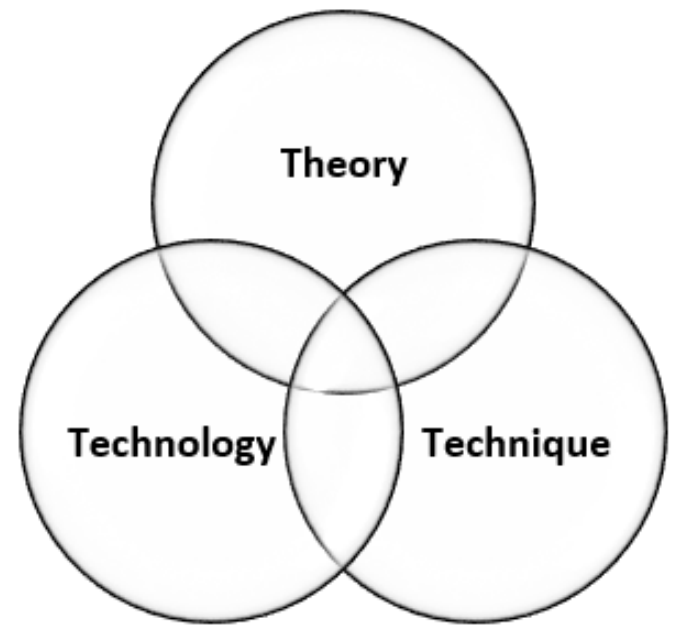

Figure 1: The overlaying area of the three major empiricism contributors constitutes the existing pure knowledge of natural phenomena

point out their interrelationships.

Therefore, a cyclic process could now be proposed via an eight-step process given in Figure 2 . The cycle contains the natural phenomena and a clear involvement of the experimenter in defining them. Each and every step of this cycle may be perceived as an individual process by itself, while each of these steps constitutes, in a sense, an individual "device" or a "gear", with a unique and particular functionality within the knowledge evolution process.

Apparently, some of these steps rely mostly on existing knowledge and experience, while others, although making use of accepted parameters, are mainly dependent on cognitional and individual interpretations of the researchers. Nonetheless, both of them will lead human decision making towards setting, defining and working on the research efficiency based on its outcome. Furthermore, experimental proficiency will now be reflected through the validation of the knowledge gaps (filled, remaining, or even opened), and the efficient use of resources for each particular research plan.

In brief, the cycle is initiated with the description of the empirically defined systemic categories which form the preservation hypothesis through the relevant physical and natural laws and their mathematical descriptions. Having established and identified such laws and relationships, the existing knowledge analyzed and logically combined, within the systemic and hypothesis boundaries, will lead to its proper classification. That then makes the experimenter capable of identifying the research gaps and selecting experimental conditions that will allow the expression of the relevant physical and natural laws' parameters. Securing or disclaiming the preservation hypothesis through this approach, will initiate an underlying new cycle for the newly refined hypothesis. This road-map is step-by-step presented and conclusively supported in Figures 2-4.

In Figure 2, each transition within the cycle of understanding represents a specific procedure towards the deep insight of the phenomenon under investigation. More precisely, for a given system, a falsifiable hypothesis must be defined (arrow 1) in order to be able to study the system under this hypothesis. To further proceed with understanding, it is now necessary to apply the relevant laws and principles (arrow 2) and to express them mathematically (arrow 3 ) before obtaining the results (arrow 4). After obtaining this information, it is necessary to obtain the cognition about the system (arrow 5) and to classify the existing knowledge (arrow 6) in order to identify the existing gaps and to design and perform new experiments (arrow 7), which will unavoidably define a new system and the cycling will now restart (arrow 8).

Consequently, in order to progress with the cycle's steps or validate and evaluate any research plan and objective, the classification of the existing knowledge is considered absolutely essential. This knowledge classification (step \#6 of Figure 2) asks for a satisfactory process that includes a thorough review of the available data and experience of the phenomena, along with a selection of both the systemic descriptors and their main participating classes. Then, knowledge could be captured within those classes-cells that have been mainly defined via the knowledge classes in the system.

In order to approach the "food-packagingenvironment system", we shall consider it as an engineering-based system following the "in-

\begin{tabular}{l|l|l|l} 
IJFS & April 2017 & Volume 6 & pages 56-66
\end{tabular} 
The distinct role of packaging on food preservation $\mid 61$

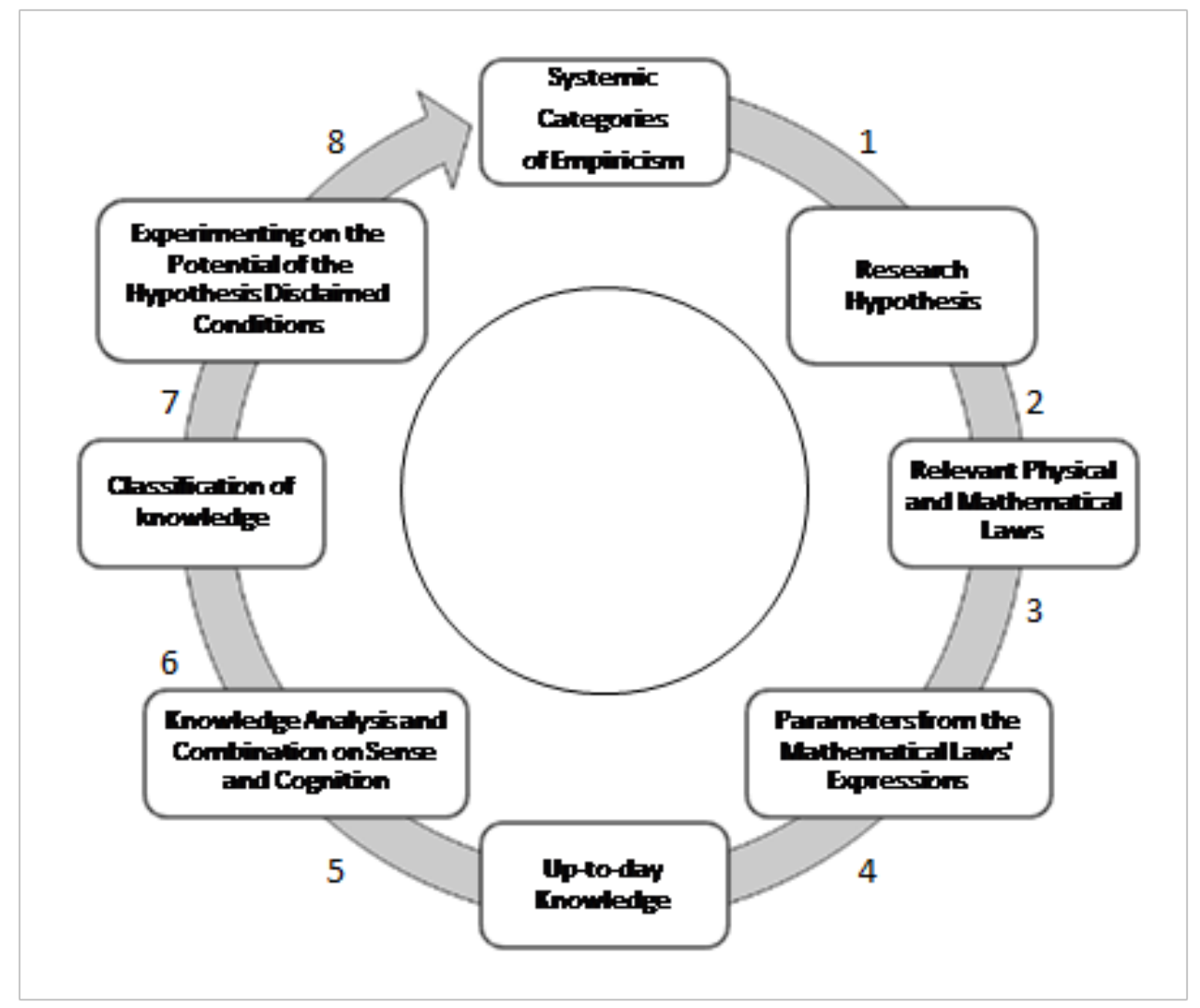

Figure 2: The cycle of understanding the physical world and increasing knowledge of physical phenomena

process-out" scheme. We may accordingly define "in" as being constituted of matter and energy, "process" as the relationships within and along the matter and energy, while "out" as the aesthetically perceived outcome. The scheme of such a system is given in Figure 3. The main knowledge classes of this scheme will be further discussed in the following section. In particular, matter, which in the case of packed food referring to all the materials constituting the system, consists of the following essential groups or knowledge classes that are actually developed with

1. each and every experience,

2. the properties,

3. the qualities, and

4. the characteristics,

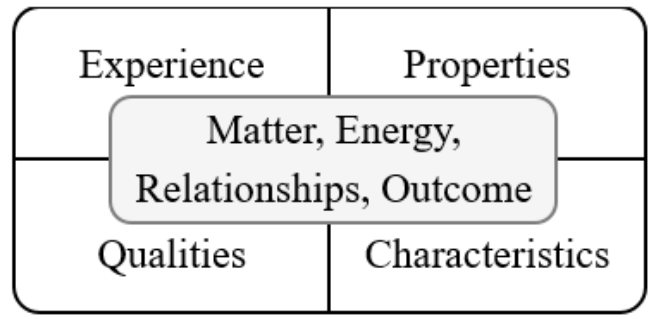

Figure 3: The four categorical descriptors of a system in the center and their main knowledge classes

for any systemic possibility in general. Accordingly, "experience" may refer to the physical dimensions; "properties" of the matter can be related to the mass transfer phenomena among systemic materials; "qualities" in a 
$62 \mid$ Kanavouras and Coutelieris

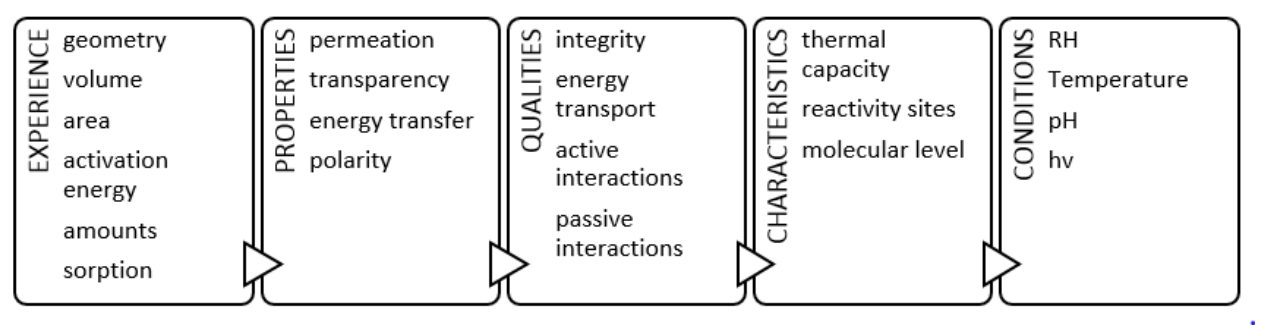

Figure 4: Exemplary accomplishment of filling the four knowledge classes and the conditions for testing the hypothesis against them

sense adhere to the indicators, markers or factors relevant to the materials; and "characteristics" propose the molecular chemistry, internal composition, recipes and concentrations of interest to the hypothesis. Based on the above scheme, experimenters essentially modify the evolution "possibilities" of the system by critically utilizing the existing knowledge, and then making cognitional decisions on how it justifiably complies with the hypothesis in question and, most importantly, the appropriateness of this knowledge. Having achieved that, innovative approaches (ideas), inventive applications (technology), practicalities (techniques) and/or alternative rationality (scientific theories) may emerge.

An assortment of contributing properties/attributes of packaging (materials, means and processes), that can be described in physical terms and measured within analytical capabilities, can potentially become model parameters for mathematical expressions. Hence, according to the aforementioned four categories and the knowledge classes within each one, a list of properties, specifications or attributes could be used to further elaborate on each and every group of "experiences", "properties", "qualities" and "characteristics" for the preservation hypothesis, regarding packaging in particular.

For a "food-packaging-environment" system that may undergo spoilage and adulterations during its preservation, the available "energy" is now discussed. This descriptor defines the progress of the phenomena within, as well as, among the elements of the matter. This descriptor may be further broken down into the experience that includes the system's energy requirements (measured empirically), the energy properties (may include energy transfer, storage, and/or systems capacity), the qualities (energy transformations within the boundaries of the system) and finally the characteristics (connecting the energy to its rate/coefficient, frequency, wavelengths and similar knowledge descriptors).

An inherent systemic uniqueness is reflected in the "relationships" among its contributors. For that, "relationships" may break down to the experience (sensed by the species reactivity), the affinity properties (of the species for each other), the qualities (such as the rate/coefficients, sequences, and/or levels of expressions) and characteristics (indicating the contributors' inter- and intra-dependencies, adequacies, deficiencies, imposing restrictions, catalytic performance etc.).

Frequently, "outcome" has been the one and only component of the collected data for a number of preservation studies. In this class, the measured experience mainly referred to is quantity, the properties of outcome are adjacent to its identification, the qualities signify the selected indicator(s), marker(s) and significance, and the characteristics could be related to the molecular chemistry of evolved compounds.

Conclusively, geometry, volume content, area, permeation, transparency, pack integrity, foodpackaging active/passive interactions, activation energy - thermal properties, energy transport, energy transfer, thermal capacity, amounts, polarity, active/passive interactions, active reactivity sites, sorption etc. are, in the opinion of the authors, essential considerations for packag- 
ing, carefully selected for the food preservation consideration, as demonstratively summarized in Figure 4.

In this Figure 4, the four main knowledge classes of the categorical descriptors of a system are presented, along with the conditions for essentially altering and controlling (engineering) the evolution "possibilities" of the system. In a sense, this last, fifth, column is the practical outcome of this method, and is of interest to the experimenters. These are the conditions under which the phenomena may be expressed or have an impact on the level of their expression. That research interest could be fully and clearly revealed via this approach, i.e. when the experimenters correlate the properties, specifications and attributes to the conditions that may impact on them. These conditions then, shall be the appropriate ones for experimentation, directly linked to the measured empiricism and from that to the knowledge classes and systemic categories. Therefore, these conditions will check, within a minimum risk, the justified packaging that shall provide the least possibility for disclaiming the food preservation hypothesis.

\section{Results and Discussion}

Verification, validation and prediction models have commonly used the outcome of certain processes, applied in systems, in order to strengthen or evaluate a hypothesis. Foreseeing phenomena and their outcome under conditions and situations that have not been empirically sensed, has also been a common practice. In that case, mathematics may become a powerful tool for not only describing but also predictively quantifying physical phenomena and their actual scales. For example, the oxidation of packaged olive oil was only understood in depth when the following steps were implemented:

1. Estimation of the chemical kinetics regarding oxidation and photo-oxidation (Coutelieris \& Kanavouras, 2005).

2. Using the above values, the development of a deterministic mass transport model including oxygen penetration through packag- ing material, diffusion and reactions, where hexanal was selected as the most appropriate descriptor of the oxidation level (Kanavouras et al., 2004; Coutelieris \& Kanavouras, 2006).

3. Using the above results for hexanal concentration within the oil phase as an adequate macroscopic quality indicator that embeds the synergistic effect of all the processes occuring during oxidation (Kanavouras \& Coutelieris, 2006, 2006).

4. The accompaniment of the above with an extensive parametric study in order to recognize the impact of each parameter on the oil's shelf-life.

In the case of olive oil oxidation, the system consists of the "oil-packaging material-environment" phases, while the hypothesis could be related to the "constantly high quality of the product". Obviously, there is a time period, after which this hypothesis is not valid any more, that needs to be defined. The principles applied for this system include mass and energy balances. Their mathematical description leads to the convective diffusion equations for each species involved and the results are usually obtained by numerically solving the system of differential equations for the species concentrations. The developed models during research and experimentation may be perceived as an outcome by itself, aiming to mathematically present the empirical knowledge by using well-known rules and criteria applied within the systemic boundaries of each hypothetical systemic world.

These results allow for a better understanding of the degradation phenomena, which enables definition of a macroscopic quality index for the system. Following that, this macroscopic index imposes new parameters and conditions that have to be investigated through a new definition of the "oil-packaging-environment" system, in respect to these parameters.

The methodology proposed in this work, provides a reliable tool, a technology platform, for assessing and comparing those factors impacting the preservation of packed foods. The tool allows the identification and perception of the research gaps, as it integrates existing knowledge collected 
and synthesized in a valuable way, via the welldefined steps of this methodology, eventually allowing for a consistent, transparent, and repeatable approach for informative and justified decisions on complex food-packaging-environment preservation problems. Hence, a research waste (in terms of resources and time) and the packaging efficiency increment could be additional benefits in using such a methodology.

Although this methodology may facilitate comparisons and knowledge interchange, it still remains on the cognition of the experimenters and developers to manage the system with caution and great concern, only to be assisted by experience and increasingly deeper understanding. Furthermore, it is worth mentioning that a research outcome after descriptors' analysis for a specific system, and the phenomena within it, may be knowledge that is similar for other systems. That conclusion could allow the interchangeably adaptation of the relevant results and knowledge during the testing of a different hypothesis.

We may additionally propose this methodological classification of data, as a knowledge storage space for maintaining, handling, preserving, monitoring and continuously evaluating developments in the particular scientific area of interest, for decision-making purposes. In that sense, the history of developments within a scientific, theoretical or practical field is captured not just as knowledge, but as impact, allowing for an explicit and critical consideration of the changes within the food matrix, the packaging materials, the processes and the environment.

Since, there are rarely adequate data and resources for a comprehensive experiment, it is common to develop predictive models projecting potential situations and their expected outcome. We support that the use of a classification methodology for knowledge, will allow for higher accuracy models, of universal applicability and broad recognition. That is so, since the classification scheme is actually a "device", a "machine" whose "operational mechanics" are only to be revealed by the full and appropriate filling of the classes' cells. Anticipated benefits from such an approach may even be extended to the educational needs and initial involvement of researchers in dissertations and experimenta- tion design of high accuracy and acceptance in applications, at least for the initial stages of a scientific hypothesis completion.

Using the knowledge classes proposed as individual areas for the preservation hypothesis disclaim possibilities, the packed food preservation sector may also create innovation scenarios and work towards a total food-packaging-environment design plan. Hence, by fulfilling the cells, we may also reveal the consequences on specific outcomes, e.g., on selected quality indicators. That shall support certain design decisions in drawing the boundaries of the system, via a self-assessment versus the preservation requirements.

Although, the methodology has proven to reveal the conditions for testing the hypothesis, it may even go a step further, by selecting the levels of the variables to be included for experimenting against potential failures. Thus, it is not only the conditions per se that are defined but also the norms of these conditions that allow for a low or high preservation risk. Furthermore, it now becomes more straightforward to define the "just-right" packaging, avoiding over- or underpackaging options.

Yet, further studies are required to better understand the potential contribution of such a method on other food or non-food related hypothesis and the experimentation on their resistance to be disclaimed. Studies in diverse scientific field may even allow for similarities among risk management techniques in a higher level of estimating processes.

\section{Conclusions}

Knowledge classification was proven useful to assist preparations for, and executions of, experiments that may lead to concrete decisions. Integration of a literature review on the phenomena cohesions, along with human cognition and empiricism, shall adequately predict and express the impacts of selected testing conditions.

Additionally, knowledge classification may act in part, as a repository of information for modeling the potential disclaim of the system's hypothesis under increased risk. Categories, descriptors, classes and specifications are to be placed within this matrix, when designing experiments

\begin{tabular}{l|l|l|l} 
IJFS & April 2017 & Volume 6 & pages 56-66
\end{tabular} 
The distinct role of packaging on food preservation $\mid 65$

and selecting packaging materials, processes and means. Therefore, we would expect with high anticipation that this methodology will allow for constructive criticism among research groups, seeking for research accuracy, minimum research waste and deeper knowledge of the phenomena. Packaging in its totality, is beyond just a passive mean for food preservation. Instead, inherent packaging properties are here to support the engineering of preservation in order to reveal the close relationship among "things" and "elements", matter and energy, quantity and quality. Packaging becomes the filter to distinguish and apply or exclude certain "conditions" to the system, a much needed step for completing our approach.

In that sense, we propose to deal with packaging procedures within the preservation hypothesis, as a "sensor" of the food and environmental impact on the hypothesis. It is packaging which, by responding to these to contributors, will to a great extent, support the persistence of the system against the preservation disclaim. Therefore, the active role (expression) of the selected packaging properties has to be defined and applied in the experimental design and set-up phase, aiming in the food degradation phenomena research, especially at the food-packaging-environment holistic level.

\section{References}

Ahrne, L. M., Oliveira, F. A. R., Manso, M. C., Drumond, M. C., Oste, R., \& Gekas, V. (1997). Modelling of dissolved oxygen concentration during storage of packaged liquid foods. Journal of Food Engineering, 34 (2), 213-224. doi:10.1016/S02608774(97)00082-4

Bacigalupi, C., Lemaistre, M. H., Boutroy, N., Bunel, C., Peyron, S., Guillard, V., \& Chalier, P. (2013). Changes in nutritional and sensory properties of orange juice packed in pet bottles: an experimental and modelling approach. Food Chemistry, 141(4), 3827-3836. doi:10.1016/j . foodchem.2013.06.076

Campos, C. A., Gerschenson, L. N., \& Flores, S. K. (2011). Development of edible films and coatings with antimicrobial activity. Food and Bioprocess Technology, 4 (6), 849 875. doi:10.1007/s11947-010-0434-1

Celia Resconi, V., Escudero, A., Antonio Beltran, J., Luis Olleta, J., Sanudo, C., \& del Mar Campo, M. (2012). Color, lipid oxidation, sensory quality, and aroma compounds of beef steaks displayed under different levels of oxygen in a modified atmosphere package. Journal of Food Science, r7y(1), S10 S18. doi:10.1111/j.1750-3841.2011.02506.x

Christophersen, A. G., Bertelsen, G., Andersen, H. J., Knuthsen, P., \& Skibsted, L. H. (1992). Storage life of frozen salmonoids - effect of light and packaging conditions on carotenoid oxidation and lipid oxidation. Zeitschrift Fur Lebensmittel - Untersuchung Und - Forschung, 194(2), 115-119. doi:10.1007/BF01190179

Coutelieris, F. A. \& Kanavouras, A. (2006). Experimental and theoretical investigation of packaged olive oil: development of a quality indicator based on mathematical predictions. Journal of Food Engineering, 73(1), 85-92. doi:10.1016/j.jfoodeng.2005.01.008

Coutelieris, F. \& Kanavouras, A. (2005). Use of the activation energy concept to estimate the quality reduction of packaged olive oil. Journal of the American Oil Chemists Society, 82(2), 119-123. doi:10.1007/s11746005-1052-y

Del Nobile, M. A., Bove, S., La Notte, E., \& Sacchi, R. (2003). Influence of packaging geometry and material properties on the oxidation kinetic of bottled virgin olive oil. Journal of Food Engineering, 57(2), 189197. doi:10.1016/S0260-8774(02)00297-2

Galic, K., Scetar, M., \& Kurek, M. (2011). The benefits of processing and packaging. Trends in Food Science \&6 Technology, 22(2-3, SI), 127-137. doi:10.1016/j.tifs . 2010.04.001

Gimenez, A., Ares, F., \& Ares, G. (2012). Sensory shelf-life estimation: a review of current methodological approaches. Food Research International, 49(1), 311-325. doi:10.1016/j.foodres.2012.07.008

Gomez, M. \& Lorenzo, J. M. (2012). Effect of packaging conditions on shelf-life of fresh 
foal meat. Meat Science, 91(4), 513-520. doi:10.1016/j.meatsci.2012.03.007

Hall, J. E. \& Otto, S. R. (1997). A study of the diffusion of oxygen in bottled food using realistic bottle shapes. Journal of Food Processing and Preservation, 21(2), 141-159. doi:10.1111/j.1745-4549.1997.tb00773.x

Jacxsens, L., Devlieghere, F., De Rudder, T., \& Debevere, J. (2000). Designing equilibrium modified atmosphere packages for fresh-cut vegetables subjected to changes in temperature. Lebensmittel-Wissenschaft UndTechnologie-Food Science and Technology, 33(3), 178-187. doi:10.1006/fstl.2000.0639

Kanavouras, A. \& Coutelieris, F. A. (2006). Shelf-life predictions for packaged olive oil based on simulations. Food Chemistry, 96(1), 48-55. doi:10.1016/j. foodchem . 2005.01.055

Kanavouras, A., Hernandez-Munoz, P., Coutelieris, F., \& Selke, S. (2004). Oxidationderived flavor compounds as quality indicators for packaged olive oil. Journal of the American Oil Chemists Society, 81(3), 251-257. doi:10.1007/s11746-004-0891-x

Kuhn, T. S. (1962). The structure of scientific revolutions (Second Edition). University of Chicago Press. Retrieved from http : / / projektintegracija . pravo . hr / _download/repository/Kuhn_Structure_of _ Scientific_Revolutions.pdf

Leygonie, C., Britz, T. J., \& Hoffman, L. C. (2011). Protein and lipid oxidative stability of fresh ostrich $\mathrm{m}$. iliofibularis packaged under different modified atmospheric packaging conditions. Food Chemistry, 127(4), 1659-1667. doi:10.1016/j.foodchem. 2011. 02.033

Mestdagh, F., De Meulenaer, B., De Clippeleer, J., Devlieghere, F., \& Huyghebaert, A. (2005). Protective influence of several packaging materials on light oxidation of milk. Journal of Dairy Science, 88(2), 499-510.

Miltz, J., Passy, N., \& Mannheim, C. H. (1992). Mass transfer from and through packaging materials. Packaging Technology and Science, 5(1), 49-56. doi:10. 1002 / pts . 2770050110

Miranda, G., Berna, A., Bon, J., \& Mulet, A. (2011). Modeling of the process of mois- ture loss during the storage of dried apricots. Food Science and Technology International, 17(5), 439-447. doi:10.1177/ 1082013211398810

Mitcham, C. (1994). Thinking through technology: the path between engineering and philosophy. University of Chicago Press.

Raso, J. \& Barbosa-Canovas, G. V. (2003). Nonthermal preservation of foods using combined processing techniques. Critical Reviews in Food Science and Nutrition, 43(3), 265-285. doi:10.1080/10408690390826527

Senorans, F. J., Ibanez, E., \& Cifuentes, A. (2003). New trends in food processing. Critical Reviews in Food Science and $\mathrm{Nu}$ trition, 43(5), 507-526. doi:10 . 1080 / 10408690390246341

Silvestre, C., Duraccio, D., \& Cimmino, S. (2011). Food packaging based on polymer nanomaterials. Progress in Polymer Science, 36(12), 1766-1782. doi:10.1016/j . progpolymsci.2011.02.003

Tsironi, T. N. \& Taoukis, P. S. (2010). Modeling microbial spoilage and quality of gilthead seabream fillets: combined effect of osmotic pretreatment, modified atmosphere packaging, and nisin on shelf life. Journal of Food Science, 75(4), M243-M251. doi:10. $1111 / \mathrm{j} .1750-3841.2010 .01574 . \mathrm{x}$

Zhou, G. H., Xu, X. L., \& Liu, Y. (2010). Preservation technologies for fresh meat - a review. Meat Science, 86(1, SI), 119-128. doi:10.1016/j.meatsci.2010.04.033

Zwietering, M., Rombouts, F., \& Van 't Riet, K. (1993). Some aspects of modelling microbial quality of food. Food Control, 4(2), 89-96. doi:10.1016/0956-7135(93)90163-I 